\title{
SUPPORTING INFORMATION \\ Protein synthesis in coupled and uncoupled cell-free prokaryotic gene expression systems
}

Maike M. K. Hansen, Marta Ventosa Rosquelles, Maaruthy Yelleswarapu, Roel J. M. Maas, Aafke J. van

Vugt-Jonker, Hans A. Heus, and Wilhelm T. S. Huck ${ }^{*}$

\section{Supporting Figures:}

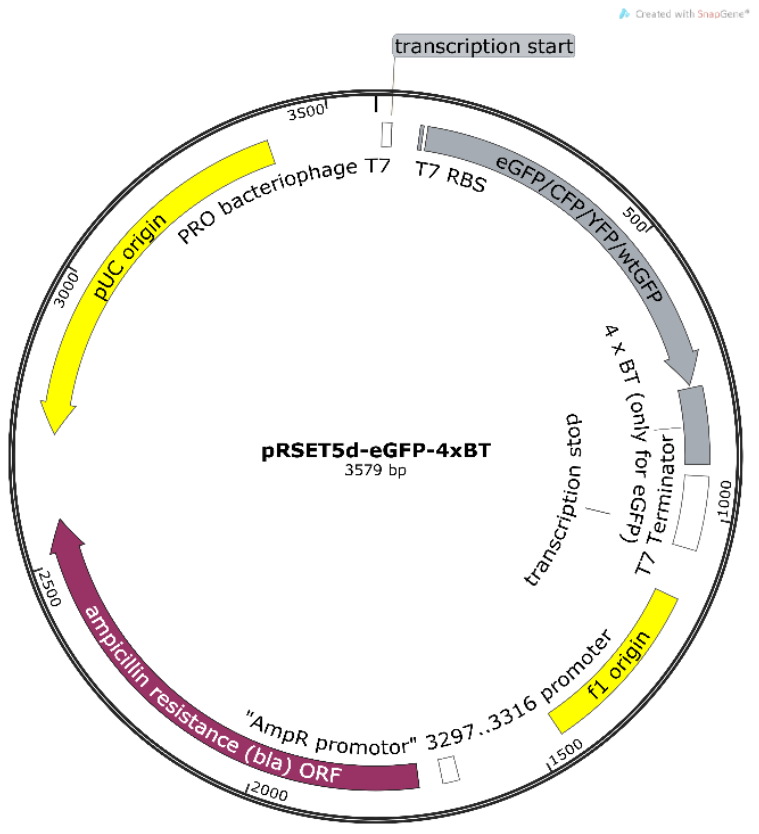

Figure S1: Plasmid used for mRNA and protein expression studies. The plasmids for YFP, CFP and eGFP4xBT all share the same backbone plasmid, namely the pRSET backbone. The plasmids containing YFP and CFP lack the 4xBT sequence.

T7 promoter-RBS-deGFP-4xBT-
T7 terminator
(BT $=$ target for molecular
beacon)
beacon)

\begin{abstract}
taatacgactcactatagggagaccacaacggtttccctctagaaataattttgtttaactttaa gaaggagatataccATGgagcttttcactggcgttgttcccatcctggtcgagctggacggcg acgtaaacggccacaagttcagcgtgtccggcgagggcgagggcgatgccacctacggcaa gctgacctgaagttcatctgcaccaccggcaagctgcccgtgccctggcccaccctcgtgacc acctgacctacggcgtgcagtgcttcagccgctacccogaccacatgaagcagcacgacttc ttcaagtccgccatgcccgaaggctacgtccaggagcgcaccatcttcttcaaggacgacggc aactacaagacccgcgccgaggtgaagttcgagggcgacacctggtgaaccgcatcgagct gaagggcatcgacttcaaggaggacggcaacatcctggggcacaagctggagtacaactac aacagccacaacgtctatatcatggccgacaagcagaagaacggcatcaaggtgaacttcaa gatccgccacaacatcgaggacggcagcgtgcagctcgccgaccactaccagcagaacacc cccatcggcgacggcccotgctgctgcccgacaaccactacctgagcacccagtccgccctg agcaaagaccccaacgagaagcgcgatcacatggtcctgctggagttcgtgaccgccgccgg gatctaactcgagtagtagaactctctcaacgtgtacgccatagctagctacaactctctcaac
\end{abstract}




\begin{tabular}{|c|c|}
\hline & $\begin{array}{l}\text { gtaacgtacctaacgcatcgaactctctcaacgttactatactaaactacctaactctctcaac } \\
\text { gtactactggaaaactacctgaattcgaagcttgatccggctgctaacaaagcccgaaagga } \\
\text { agctgagttggctgctgccaccgctgagcaataactagcataaccccttggggcctctaaacg } \\
\text { ggtcttgaggggttttttgctgaaaggaggaactatatccggat }\end{array}$ \\
\hline wtGFP & $\begin{array}{l}\text { ATGggtaaaggagaagaacttttcactggagttgtcccaattcttgttgaattagatggtgat } \\
\text { gttaatgggcacaaattttctgtcagtggagagggtgaaggtgatgcaacatacggaaaactt } \\
\text { acccttaaatttatttgcactactggaaaactacctgttccgtggccaacacttgtcactactttc } \\
\text { tcttatggtgttcaatgcttttcccgttatccggatcatatgaaacggcatgactttttcaagagt } \\
\text { gccatgcccgaaggttatgtacaggaacgcactatatctttcaaagatgacgggaactacaag } \\
\text { acgcgtgctgaagtcaagtttgaaggtgatacccttgttaatcgtatcgagttaaaaggtattg } \\
\text { attttaaagaagatggaaacattctcggacacaaactcgagtacaactataactcacacaatg } \\
\text { tatacatcacggcagacaaacaaaagaatggaatcaaagctaacttcaaaattcgccacaac } \\
\text { attgaagatggatccgttcaactagcagaccattatcaacaaaatactccaattggcgatggc } \\
\text { cctgtccttttaccagacaaccattacctgtccacacaatctgccctttcgaaagatcccaacga } \\
\text { aaagagagaccacatggtccttcttgagtttgtaacagctgctgggattacacatggcatggat } \\
\text { gaactatacaagggtcaccaccatcaccatcactag }\end{array}$ \\
\hline CFP & $\begin{array}{l}\text { ATGgtgagcaagggcgaggagctgttcaccggggtggtgcccatcctggtcgagctggacg } \\
\text { gcgacgtaaacggccacaggttcagcgtgtccggcgagggcgagggcgatgccacctacggc } \\
\text { aagctgaccctgaagttcatctgcaccaccggcaagctgcccgtgccctggcccaccctcgtg } \\
\text { accaccctgacctggggcgtgcagtgcttcagccgctaccccgaccacatgaagcagcacga } \\
\text { cttcttcaagtccgccatgcccgaaggctacgtccaggagcgtaccatcttcttcaaggacgac } \\
\text { ggcaactacaagacccgcgccgaggtgaagttcgagggcgacaccctggtgaaccgcatcg } \\
\text { agctgaagggcatcgacttcaaggaggacggcaacatcctggggcacaagctggagtacaa } \\
\text { ctacatcagccacaacgtctatatcaccgccgacaagcagaagaacggcatcaaggcccact } \\
\text { tcaagatccgccacaacatcgaggacggcagcgtgcagctcgccgaccactaccagcagaac } \\
\text { acccccatcggcgacggccccgtgctgctgcccgacaaccactacctgagcacccagtccaag } \\
\text { ctgagcaaagaccccaacgagaagcgcgatcacatggtcctgctggagttcgtgaccgccgc } \\
\text { cgggatcactcacggcatggacgagctgtacaaggctggtaagcttgcggccgcactcgagc } \\
\text { aagaattcgaagcttga }\end{array}$ \\
\hline YFP & $\begin{array}{l}\text { ATGgtgagcaagggcgaggagctgttcaccggggtggtgcccatcctggtcgagctggacg } \\
\text { gcgacgtaaacggccacaagttcagcgtgtccggcgagggcgagggcgatgccacctacggc } \\
\text { aagctgaccctgaagttcatctgcaccaccggcaagctgcccgtgccctggcccaccctcgtg } \\
\text { accaccttcggctacggcctgaagtgcttcgcccgctaccccgaccacatgaagcagcacgac } \\
\text { ttcttcaagtccgccatgcccgaaggctacgtccaggagcgcaccatcttcttcaaggacgacg } \\
\text { gcaactacaagacccgcgccgaggtgaagttcgagggcgacaccctggtgaaccgcatcga } \\
\text { gctgaagggcatcgacttcaaggaggacggcaacatcctggggcacaagctggagtacaact } \\
\text { acaacagccacaacgtctatatcatggccgacaagcagaagaacggcatcaaggtgaacttc } \\
\text { aagatccgccacaacatcgaggacggcagcgtgcagctcgccgaccactaccagcagaaca } \\
\text { cccccatcggcgacggccccgtgctgctgcccgacaaccactacctgagctaccagtccaagc } \\
\text { tgagcaaagaccccaacgagaagcgcgatcacatggtcctgctggagttcgtgaccgccgcc } \\
\text { gggatcactcacggcatggacgagctgtacaaggctggtaagcttgcggccgcactcgagca } \\
\text { agaattcgaagcttga }\end{array}$ \\
\hline
\end{tabular}




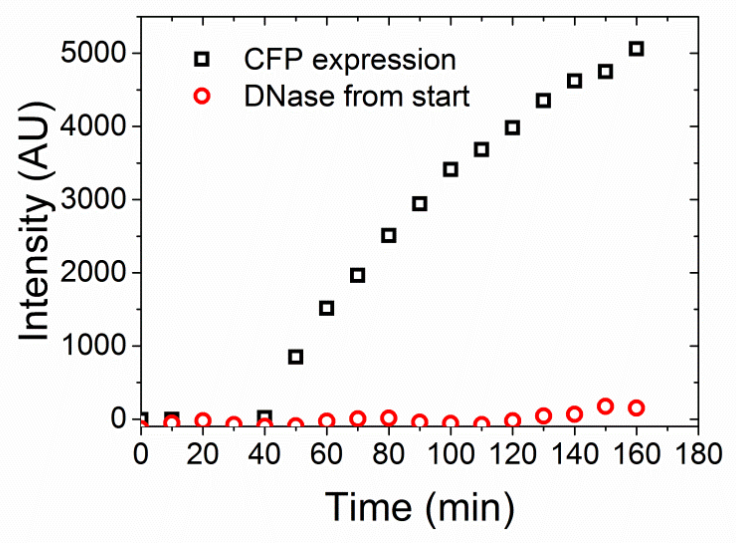

Figure S2: Addition of DNase in a transcription system from time 0. Here we clearly see no CFP expression meaning that DNase fully arrests gene expression.
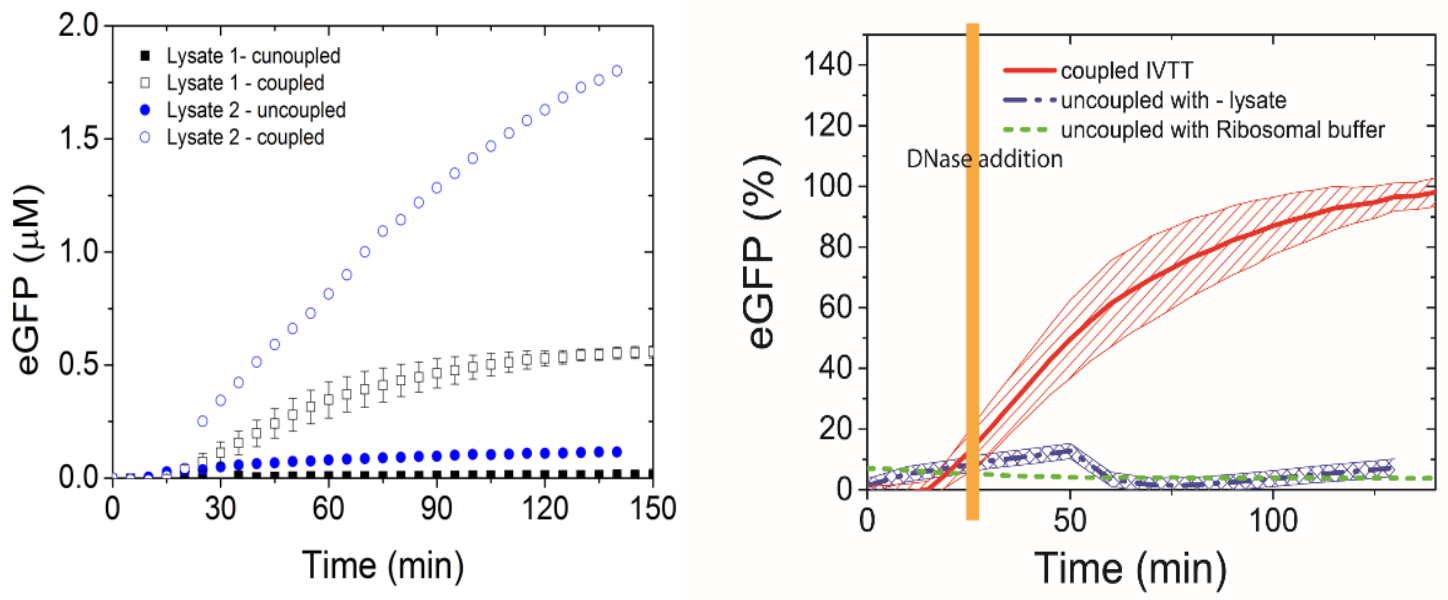

Figure S3: Left: Uncoupled protein expression vs coupled protein expression with two different lysates. Different lysate batches, which express proteins at different yields, still show similar ratio between coupled and uncoupled gene expression. Right: Uncoupling in the presence of lysate without ribosomes (-lysate), and in the presence of ribosomal extract buffer (STB). Coupled protein expression is shown by the red full line. Uncoupled gene expression in the presence of lysate without ribosomes (-lysate) is shown by the blue dashed-dotted line, the -lysate was obtained by dialysing the supernatant of the ribosomal extract, see methods for more detail. In the presence of ribosomal extract buffer, i.e. the buffer where the ribosomal extract is stored in, (green dashed line) there was no protein expression. Since uncoupling gene expression in the presence of lysate without ribosomes (-lysate), and in the presence of ribosome buffer do not yield any protein, it is a strong indication that the ribosomal extract rescues the protein expression. 

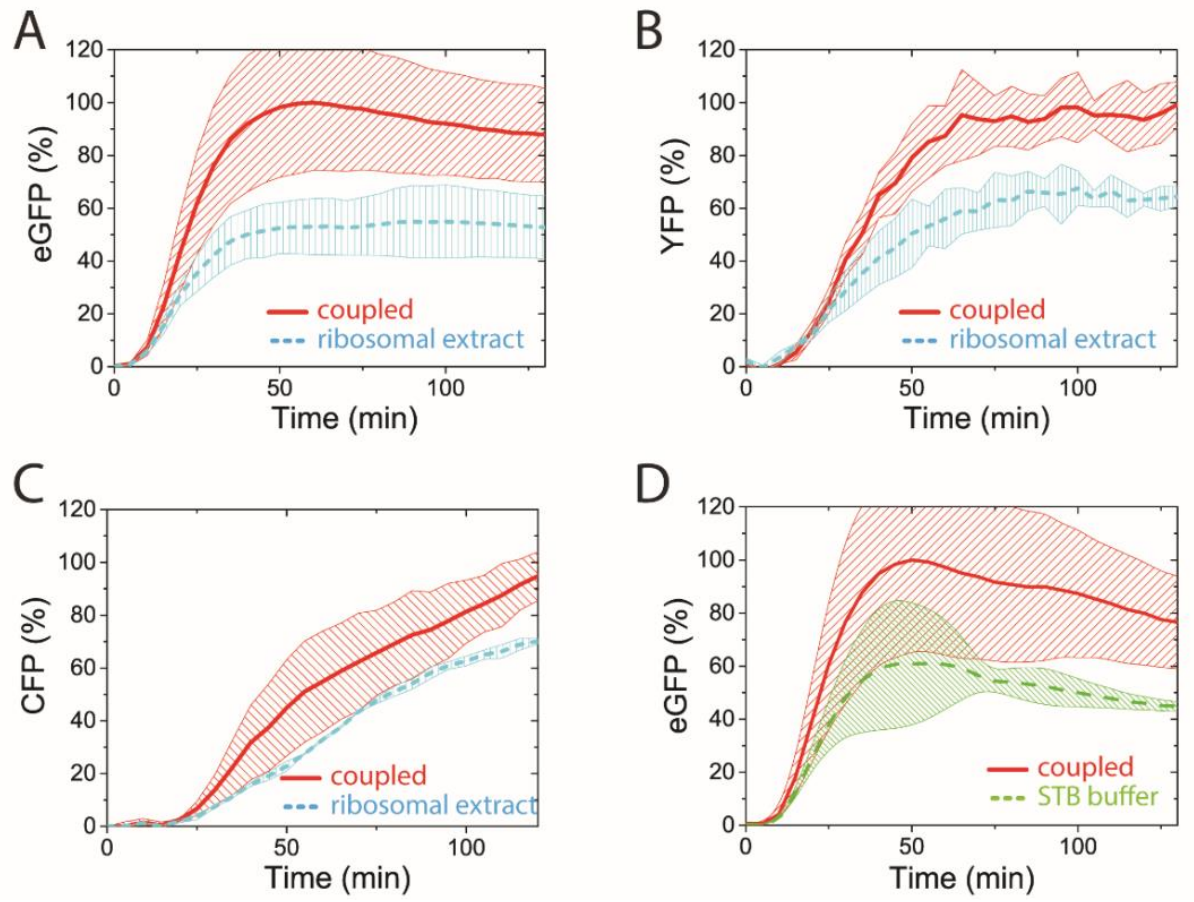

Figure S4. The addition of ribosomal extract decreases total expression yield for (A) eGFP, (B) YFP and (C) CFP, the red solid line shows normal coupled in vitro transcription and the cyan dashed line shows coupled IVTT in the presence of additional $5 \mu \mathrm{M}$ of ribosomal extract. (D) Coupled gene expression in the presence of the ribosome buffer.

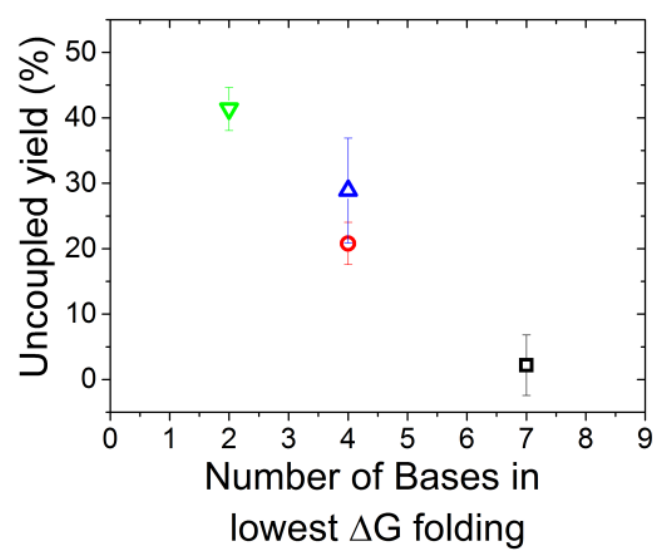

Figure S5: Secondary structure predictions for a 50 base pair region encompassing the RBS and start codon, wtGFP (green triangle), CFP and YFP (blue triangle and red circle respectively) and eGFP (black square). In Figure $3 \mathrm{~d}$ in the main text, all structure predictions performed, started at the same position within the respective plasmids. Here, we determined number of bases involved in secondary structure for similar total free energy values (-4.6 for eGFP/wtGFP and -4.5 for CFP/YFP), meaning the starting point of the 50 base pair region could vary per plasmid. Again, a clear linear relationship between bases involved in folding and protein yield can be observed. The only difference compared to Figure $3 \mathrm{~d}$, is that eGFP has 7 bases in involved in the lowest energy fold instead of 8 . 

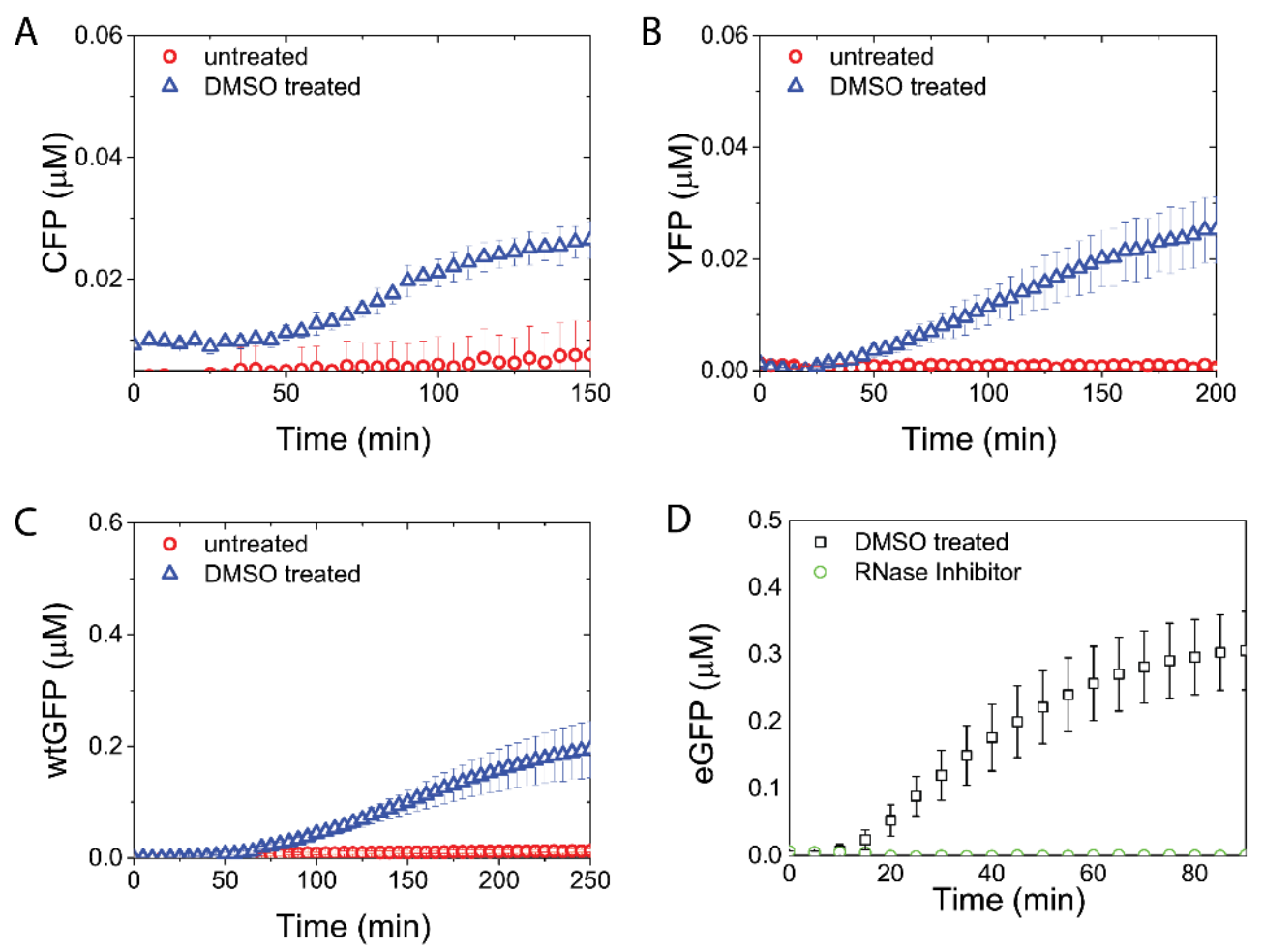

Figure S6: in vitro translation of DMSO treated mRNA for YFP (A), CFP (B) and wtGFP (C). Comparing DMSO treated (blue triangles) and untreated (red circles) mRNA the DMSO treatment clearly enhances translation for all proteins. (D) RNase inhibitors (1U/ $/ \mathrm{L}$, human placental from NEB) was added to the in vitro translation mixture (green circles) and compared to expression from DMSO treated mRNA (black squares).

\section{Supporting Methods:}

\section{The cell lysate (34 $\mathrm{mg} \mathrm{mL}^{-1}$ ) for the DMSO experiments was prepared as follows:}

A starter culture of E.coli Rosetta2 was prepared in 2xYT broth (16 g tryptone, $10 \mathrm{~g}$ yeast extract, $5 \mathrm{~g}$ $\mathrm{NaCl}$ dissolved in $850 \mathrm{~mL}$ water and autoclaved) and cultured at $37^{\circ} \mathrm{C}$ overnight. To $850 \mathrm{~mL}$ of autoclaved 2YT broth, $50 \mathrm{~mL}$ of starter culture was added along with $100 \mathrm{~mL}$ of $10 \mathrm{x}$ 2YPTG salt $(0.22$ $\mathrm{M} \mathrm{NaH}_{2} \mathrm{PO}_{4}, 0.55 \mathrm{M} \mathrm{Na}_{2} \mathrm{HPO}_{4}$, titrated to $\mathrm{pH} 7.0$, autoclaved) and $18.02 \mathrm{~g}$ glucose and incubated on a shaker $(230-250 \mathrm{rpm})$ at $37^{\circ} \mathrm{C} . \mathrm{OD}_{600}$ was checked regularly and the culture was harvested at $\mathrm{OD}_{600}=$ 1.8. The cells were collected by centrifugation at $5500 \mathrm{rpm}$ for $10 \mathrm{mins}$ at $4^{\circ} \mathrm{C}$ in three JA-10 vials. The pellets were re-suspended in ice-cold lysis buffer (20\% sucrose, $1 \mathrm{mM}$ EDTA, $1 \mathrm{mg} \mathrm{mL}^{-1}$ egg white lysozyme, filter-sterilised, $16 \mathrm{~mL} / \mathrm{JA}-10$ vial) and incubated for $10 \mathrm{mins}$ on ice. The dissolved pellets were pooled and $24 \mathrm{~mL}$ of ice-cold MQ water was added. They were incubated for 5 more minutes on 
ice and centrifuged at $7500 \mathrm{rpm}$ for 10 mins at $4^{\circ} \mathrm{C}$. The supernatant was drained and the pellet was weighed. The pellet was resuspended in ice-cold milliQ water $(0.75 \mathrm{x}$ volume) carefully. The cells were lysed by 10 cycles of 10 seconds of sonication at 10uM amplitude with 30 s breaks on ice between cycles. Once sonicated, the cells were centrifuged at $10000 \mathrm{rpm}$ for $10 \mathrm{mins}$ at $4^{\circ} \mathrm{C}$. The supernatant was collected, incubated at $37^{\circ} \mathrm{C}$ for 30 minutes and centrifuged again at $15700 \mathrm{rpm}$ at $4^{\circ} \mathrm{C}$ for $10 \mathrm{mins}$. The supernatant, now lysate, was dialysed against $50 \%$ dialysis buffer $(5 \mathrm{mM}$ Tris-Cl, 30mM potassium glutamate, $7 \mathrm{mM}$ magnesium glutamate, $0.5 \mathrm{mM}$ DTT, autoclaved) for 45 minutes followed by $3 \times 45$ minutes dialysis against $100 \%$ dialysis buffer $(10 \mathrm{mM}$ Tris-Cl, $60 \mathrm{mM}$ potassium glutamate, $14 \mathrm{mM}$ magnesium glutamate, $1 \mathrm{mM}$ DTT, autoclaved). The lysate was aliquoted and flash-frozen in liquid nitrogen and stored at $-80^{\circ} \mathrm{C}$. Protein concentration was determined using a Pierce ${ }^{\mathrm{TM}} \mathrm{BCA}$ Protein Assay Kit. 